\title{
Continued-fraction expansion of eigenvalues of generalized evolution operators in terms of periodic orbits
}

\author{
Hirokazu Fujisaka ${ }^{1}$, Hideto Shigematsu ${ }^{1}$ and Bruno Eckhardt ${ }^{2}$ \\ 1 Department of Physics, Kyushu University 33, Fukuoka 812, Japan \\ 2 Fachbereich Physik der Philipps-Universität, D-3550 Marburg, \\ and \\ FB 8 and ICBM, C.v. Ossietzky Universität, Postfach 2503, D-2900 Oldenburg \\ Federal Republic of Germany
}

\begin{abstract}
A new expansion scheme to evaluate the eigenvalues of the generalized evolution operator (Frobenius-Perron operator) $H_{q}$ relevant to the fluctuation spectrum and poles of the order- $q$ power spectrum is proposed. The "partition function" is computed in terms of unstable periodic orbits and then used in a finite pole approximation of the continued fraction expansion for the evolution operator. A solvable example is presented and the approximate and exact results are compared; good agreement is found.
\end{abstract}

PACS 05.45.+b 


\section{Introduction}

The hallmark of chaos is the exponential growth of initial uncertainty, i.e., the sensitivity on initial condition. This stems from the fact that all periodic orbits are unstable, and the representative point wanders over the state space. As a result the dynamical behavior of the system is quite irregular. This is the fundamental reason for the necessity of a statistical description of chaotic systems.

It is interesting to know, however, that despite of their instability, many statistical quantities are well approximated by periodic orbits. The periodic-orbit approximation of the natural invariant measure has been introduced by Sinai, Bowen, Ruelle in the 1970's [1] in the context of hyperbolic systems. Cycles have then been used for the characterization of strange sets and local expansion rates [2] and for the calculation of damping rates in the conventional double time correlation function [3]. The main purpose of the present paper is to show how fluctuation and order- $q$ power spectra, which have previously been introduced to describe the long-time statistics and the overall temporal correlations of chaotic fluctuations [4-7], can be calculated using periodic orbits and the continued-fraction expansion $[6,8,9]$. We will thus obtain a new expansion scheme for the eigenvalues of the generalized Frobenius-Perron operator $H_{q}$, which plays an important role for the statistical description of chaotic fluctuations.

The present paper is organized as follows. In section 2 we give a brief review of our approach to dynamical fluctuations. In section 3 we will derive fundamental equations which determine the eigenvalues in terms of unstable periodic orbits; they generalize the ones studied in Ref. 3. A new scheme for the estimation of the eigenvalues is proposed in section 4 by using the continued-fraction expansion. A solvable non-obvious example is 
presented in section 5, and finite-pole approximations of the continued-fraction expansion are compared with the exact results. The paper is closed in section 6 with some final remarks. The relation between the generating function and the generalized evolution operator and the cycle expansion of the Fredholm determinant are discussed in appendices.

\section{Statistical characterization of dynamical fluctuations}

Consider a dissipative, chaotic $d$-dimensional map

$$
\boldsymbol{x}(j+1)=\boldsymbol{f}(\boldsymbol{x}(j)), \quad \boldsymbol{x}=\left(x_{1}, x_{2}, \cdots, x_{d}\right) .
$$

Let $u_{j}=u\{\boldsymbol{x}(j)\}$ be an observed time series which is a unique, scalar function of $\boldsymbol{x}(j)$ generated by (2.1). The probability distribution $p_{n}(u)$ that the average

$$
\bar{u}_{n}(\boldsymbol{x}(0))=\frac{1}{n} \sum_{j=0}^{n-1} u\{\boldsymbol{x}(j)\},
$$

takes a value $u$ is asymptotically given by $[4,6,10]$

$$
\begin{aligned}
p_{n}(u) & \equiv<\delta\left(\bar{u}_{n}-u\right)> \\
& =\int \delta(\bar{u}(\boldsymbol{x})-u) \rho_{*}(\boldsymbol{x}) d x \\
& \sim e^{-s(u) n},
\end{aligned}
$$

for large $n$. Here $\rho_{*}(\boldsymbol{x})$ is the natural invariant density, satisfying

$$
\rho_{*}(\boldsymbol{x})=\int \delta(\boldsymbol{x}-\boldsymbol{f}(\boldsymbol{y})) \rho_{*}(\boldsymbol{y}) d \boldsymbol{y} \equiv H \rho_{*}(\boldsymbol{x}),
$$

with the Frobenius-Perron (FP) operator $H$. The generating function

$$
M_{q}(n)=<\exp \left[q \sum_{j=0}^{n-1} u\{\boldsymbol{x}(j)\}\right]>
$$


asymptotically takes the form

$$
M_{q}(n) \sim e^{\phi(q) n}
$$

for large $n$. The functions $\phi$ and $s$ are related to each other via $[4,6,10]$

$$
\phi(q)=-\min _{u}[s(u)-q u]
$$

The function $\phi(q)$ plays a fundamental role for the overall characterization of temporal fluctuations. However it cannot describe the explicit temporal correlations in $\{u\{\boldsymbol{x}(j)\}\}$. These can be singled out as follows. Let us define a weighted average

$$
\begin{aligned}
u(q) & =d \phi(q) / d q \\
& =\lim _{n \rightarrow \infty}\left\langle\bar{u}(\boldsymbol{x}(0)) e^{q n \bar{u}_{n}(\mathbf{x}(0))}\right\rangle / M_{q}(n),
\end{aligned}
$$

and the order- $q$ power spectrum [7]

$$
\begin{aligned}
I_{q}(\omega) & =\lim _{n \rightarrow \infty}\left\langle F_{n}(\omega, \boldsymbol{x}(0)) \delta\left(\bar{u}_{n}(\boldsymbol{x}(0))-u(q)\right)\right\rangle / p_{n}(u(q)) \\
& =\lim _{n \rightarrow \infty}\left\langle F_{n}(\omega, \boldsymbol{x}(0)) e^{q n \bar{u}_{n}(\mathbf{x}(0))}\right\rangle / M_{q}(n),
\end{aligned}
$$

where

$$
F_{n}(\omega, \boldsymbol{x}) \equiv \frac{1}{n}\left|\sum_{j=0}^{n-1}\left[u\left\{\boldsymbol{f}^{j}(\boldsymbol{x})\right\}-u(q)\right] e^{-i \omega j}\right|^{2} .
$$

Then $I_{q}(\omega)$ is identical to the power spectrum over time regions where the coarse-grained quantities $\bar{u}_{n}$ take the same value $u(q)$ and singles out the temporal correlation characteristic of the intensive parameter $q$. In addition, one can define the generalized double time correlation function $C_{q}(n)$ by an ensemble average as above. It turns out that $I_{q}(\omega)$ and $C_{q}(n)$ are related to each other via the Wiener-Khinchin theorem.

If there exist only poles, one obtains [7]

$$
I_{q}(\omega)=\frac{1}{2} \sum_{l}^{\prime} K_{q}^{(l)} \frac{\sinh \left[\gamma_{q}^{(l)}+i \omega_{q}^{(l)}\right]}{\sinh ^{2}\left[\frac{\gamma_{q}^{(l)}+i \omega_{q}^{(l)}}{2}\right]+\sin ^{2}\left(\frac{\omega}{2}\right)},
$$


and the corresponding order- $q$ double time correlation function takes the form

$$
C_{q}(n)=\sum_{l}^{\prime} K_{q}^{(l)} \exp \left[-\left\{\gamma_{q}^{(l)}+i \omega_{q}\right\} n\right]
$$

where $\left\{\gamma_{q}^{(l)}\right\}$ and $\left\{\omega_{q}^{(l)}\right\}$ are respectively the sets of decay rates and characteristic frequencies. The ordinary power spectrum [11] is given by $I_{q=0}(\omega)[7]$.

\section{Determination of eigenvalues of $H_{q}$ by periodic cycles}

In appendix A (see also [5]), we show that eq. (2.5) can be rewritten as

$$
M_{q}(n)=\int H_{q}^{n} \rho_{*}(\boldsymbol{x}) d \boldsymbol{x}
$$

where $H_{q}$ is the generalized Frobenius-Perron (GFP) operator defined by $[5,12]$

$$
H_{q} F(\boldsymbol{x})=\int \delta(\boldsymbol{x}-\boldsymbol{f}(\boldsymbol{y})) e^{q u\{\mathbf{y}\}} F(\boldsymbol{y}) d \boldsymbol{y}
$$

$H_{0}$ is identical to the usual FP operator. Let $\nu_{q}^{(l)}$ and $\psi_{q}^{(l)}$ be the eigenvalues and eigenfunctions of $H_{q}$,

$$
H_{q} \psi_{q}^{(l)}(\boldsymbol{x})=\nu_{q}^{(l)} \psi_{q}^{(l)}(\boldsymbol{x})
$$

Expanding $\rho_{*}(\boldsymbol{x})$ in terms of $\left\{\psi_{q}^{(l)}(\boldsymbol{x})\right\}$, and inserting it into (3.1) we obtain

$$
M_{q}(n)=\sum_{l} J_{q}^{(l)}\left[\nu_{q}^{(l)}\right]^{n}
$$

with expansion coefficients $\left\{J_{q}^{(l)}\right\},\left(\sum_{l} J_{q}^{(l)}=1\right)$. Here and throughout the paper we assume that $H_{q}$ has only discrete eigenvalues. Therefore, the characteristic function $\phi(q)$ is determined by the largest eigenvalue $\nu_{q}^{(0)}$, which is positive because of the Perron theorem, viz.

$$
\phi(q)=\log \nu_{q}^{(0)}
$$


$\left(\nu_{0}^{(0)}=1\right)$

The order- $q$ power spectrum and the double time correlation function can be expressed as in (2.11), the poles being determined by

$$
\exp \left[\gamma_{q}^{(l)}+i \omega_{q}^{(l)}\right]=\nu_{q}^{(0)} / \nu_{q}^{(l)}, \quad(l \neq 0)
$$

The coefficients in (2.11) are obtained from [7]

$$
\begin{aligned}
K_{q}^{(l)} & =a_{q}(0, l) a_{q}(l, 0) \\
u\{\boldsymbol{x}\} \psi_{q}^{(l)}(\boldsymbol{x}) & =\sum_{l^{\prime}} a_{q}\left(l, l^{\prime}\right) \psi_{q}^{\left(l^{\prime}\right)}(\boldsymbol{x}),
\end{aligned}
$$

assuming completeness of the eigenfunctions. The functions $I_{q}(\omega)$ and $C_{q}(n)$ concern the explicit temporal correlation as singled out with the parameter value $q$ from infinitely many aspects of temporal-correlation characteristics embedded in $\{\boldsymbol{u}\{\boldsymbol{x}(j)\}\}$. The ordinary double time correlation function $C(n) \equiv\langle\delta u\{\boldsymbol{x}(n)\} \delta u\{\boldsymbol{x}(0)\}\rangle$, (where $\delta u=u-\langle u\rangle)$, simply describes only one form, corresponding to the limit $q \rightarrow 0,\left(C(n)=C_{0}(n)\right)$.

Let us define the $\boldsymbol{x} \boldsymbol{y}$ element of the operator $H_{q}$ by

$$
\left(H_{q}\right)_{\mathbf{x y}} \equiv \delta(\boldsymbol{x}-\boldsymbol{f}(\boldsymbol{y})) e^{q u\{\mathbf{y}\}},
$$

and therefore

$$
\left(H_{q}^{n}\right)_{\mathbf{x y}}=\delta\left(\boldsymbol{x}-\boldsymbol{f}^{n}(\boldsymbol{y})\right) \exp \left[q \sum_{j=0}^{n-1} u\left\{\boldsymbol{f}^{j}(\boldsymbol{y})\right\}\right] .
$$

The partition function

$$
Z_{q}(n) \equiv \operatorname{Tr} H_{q}^{n}=\int\left(H_{q}^{n}\right)_{\mathbf{x x}} d \boldsymbol{x}
$$

is expanded as

$$
\begin{gathered}
Z_{q}(n)=\int \delta\left(\boldsymbol{x}-\boldsymbol{f}^{n}(\boldsymbol{x})\right) \exp \left[q \sum_{j=0}^{n-1} u\left\{\boldsymbol{f}^{j}(\boldsymbol{x})\right\}\right] d \boldsymbol{x} \\
=\sum_{k} \frac{\exp \left[q \sum_{j=0}^{n-1} u\left\{\boldsymbol{f}^{j}\left(\boldsymbol{x}^{(n), k}\right)\right\}\right]}{\left|\operatorname{det}\left[\hat{1}-\hat{D}_{n}\left(\boldsymbol{x}^{(n), k}\right)\right]\right|} . \\
-6-
\end{gathered}
$$


Here the summation $\sum_{k}$ extends over all fixed points $\left\{\boldsymbol{x}^{(n), k}\right\}$, where $\boldsymbol{x}^{(n), k}$ denotes the $k$-th fixed point of the $n$-th iterate $\boldsymbol{f}^{n}(\boldsymbol{x}),(k=1,2, \cdots, n)$,

$$
\boldsymbol{x}^{(n), k}=\boldsymbol{f}^{n}\left(\boldsymbol{x}^{(n), k}\right)
$$

Furthermore $\left(\hat{D}_{n}(\boldsymbol{x})\right)_{j k}=\partial\left(\boldsymbol{f}^{n}(\boldsymbol{x})\right)_{j} / \partial x_{k}$, i.e.,

$$
\hat{D}_{n}(\boldsymbol{x})=\prod_{j=0}^{n-1}+\hat{g}\left(\boldsymbol{f}^{j}(\boldsymbol{x})\right), \quad(\hat{g}(\boldsymbol{x}))_{k l}=\frac{\partial f_{k}(\boldsymbol{x})}{\partial x_{l}}
$$

$\prod_{+}$being the ordered product.

The spectral decomposition of $H_{q}$ on the other hand yields

$$
\operatorname{Tr} H_{q}^{n}=\sum_{l}\left[\nu_{q}^{(l)}\right]^{n}
$$

Together with (3.11) we thus obtain a set of fundamental relations between the eigenvalues $\left\{\nu_{q}^{(l)}\right\}$ and the periodic cycles as contained in $Z_{q}(n)$, viz.

$$
\sum_{l}\left[\nu_{q}^{(l)}\right]^{n}=Z_{q}(n)
$$

$(n=1,2,3, \cdots)$. The next problem is to solve for the $\left\{\nu_{q}^{(l)}\right\}$ in terms of $\left\{Z_{q}(n)\right\}$ which are determined by periodic orbits as in (3.11). In Ref. [3] and appendix B, the cycle expansion method is used for this purpose. Our main aim here is to study the applicability of a continued fraction expansion, to which we turn now.

\section{Continued-fraction expansion for the eigenvalues}

Let $A[\mu]$ denote the Laplace transform of a function $A(n)$,

$$
A[\mu] \equiv \sum_{n=1}^{\infty} A(n) \mu^{n-1} .
$$


Applied to eq.(3.15) one finds

$$
Z_{q}[\mu]=\sum_{l}\left(\frac{1}{\nu_{q}^{(l)}}-\mu\right)^{-1} .
$$

The eigenvalues $\left\{\nu_{q}^{(l)}\right\}$ are thus determined by the poles of $Z_{q}[\mu]$, which we estimate from a continued fraction expansion.

For convenience, let us put

$$
(n)_{1} \equiv Z_{q}(n)
$$

$(n=1,2,3, \cdots)$. Let $(n)_{1}$ obey the "equation of motion",

$$
(n+1)_{1}=b_{1}(n)_{1}+\sum_{j=1}^{n-1}(n-j)_{2}(j)_{1},
$$

where $b_{1}=(2)_{1} /(1)_{1}$. In the memory term a new function $(n)_{2}$ appears. By constructing an equation of motion for $(n)_{2}$ similarly to (4.4), a new function $(n)_{3}$ enters. Repeating this procedure successively, we have a set of functions $\left\{(n)_{k}, k=1,2, \cdots\right\}$, related to each other via

$$
(n+1)_{k}=b_{k}(n)_{k}+\sum_{j=1}^{n-1}(n-j)_{k+1}(j)_{k},
$$

where $b_{k} \equiv(2)_{k} /(1)_{k},(k=1,2, \cdots)$. Equation (4.5) is regarded as the equation of motion for $(n)_{k}$ determined by the memory kernel $(n)_{k+1}$.

The Laplace transform of $(n)_{k}$ is given by

$$
\begin{aligned}
{[\mu]_{k} } & \equiv \sum_{n=1}^{\infty}(n)_{k} \mu^{n-1}, \\
& =\frac{(1)_{k}}{1-b_{k} \mu-[\mu]_{k+1} \mu^{2}} .
\end{aligned}
$$

Thus we get the continued-fraction expansion [6]

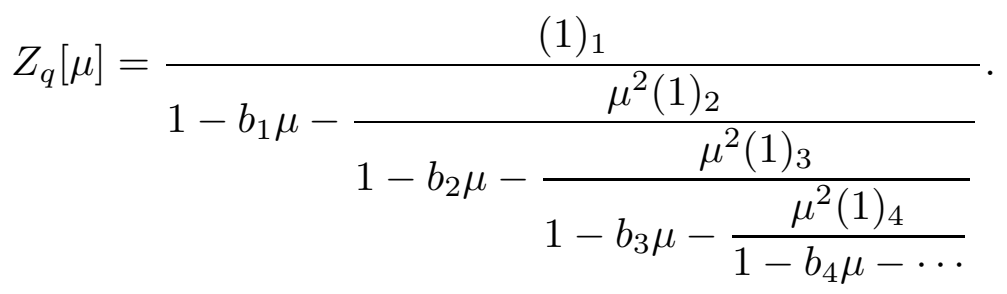


Since $\left\{\nu_{q}^{(l)}\right\}$ are identical to the reciprocal poles of $Z_{q}[\mu]$, we find

$$
\nu_{q}^{(l)}=1 / \mu_{q}^{(l)}
$$

where $\left\{\mu_{q}^{(l)}\right\}$ are poles of (4.7), i.e., the zeros of the denominator. Obviously, the continuedfraction representation (4.7) can be expanded as (4.2).

Now let us turn to how to determine the coefficients $\left\{(1)_{k}, b_{k}, k=2,3,4, \cdots\right\}$ in $(4.7)$. One should note that the quantity $(n)_{k+1}$ is determined from $\left\{(j)_{k}, j=1,2, \cdots, n+2\right\}$ by

$$
(n)_{k+1}=\frac{1}{(1)_{k}}\left\{(n+2)_{k}-b_{k}(n+1)_{k}-\sum_{l=1}^{n-1}(l)_{k+1}(n+1-l)_{k}\right\}
$$

with

$$
(1)_{k+1}=\frac{1}{(1)_{k}}\left\{(3)_{k}-b_{k}(2)_{k}\right\}
$$

$(k=1,2, \cdots)$. So the quantity $(n)_{k}$ is given in terms of $\left\{(j)_{1}, j=1,2, \cdots, n+2(k-1)\right\}$. Especially, parameters $(1)_{k}$ and $(2)_{k},(k=2,3,4, \cdots)$, in (4.7) are given, respectively, by sets $\left\{Z_{q}(j), j=1,2, \cdots, 2 k-1\right\}$ and $\left\{Z_{q}(j), j=1,2, \cdots, 2 k\right\}$. How $(1)_{k}$ and $(2)_{k}$ are determined by $\left\{Z_{q}(j)\right\}$ is given by solving the recursion relation (4.9) with the initial condition (4.10).

Since from a practical viewpoint the infinite continued-fraction expansion is intractable, we must develop a discard approximation. This can be carried out with the finitepole approximation of the continued-fraction expansion as follows. Let us assume that the eigenvalues are ordered, $\nu_{q}^{(0)}>\left|\nu_{q}^{(1)}\right| \geq\left|\nu_{q}^{(2)}\right| \geq \cdots$, and let us terminate (3.15) after $m$ terms, i.e.,

$$
\sum_{l=0}^{m-1}\left[\nu_{q}^{(l)}\right]^{n}=Z_{q}(n)
$$


This is reasonable, if the eigenvalues with $l \leq m$ decay sufficiently rapidly. This is equivalent to the $m$-pole approximation $(1)_{m+1}=0, i . e .$,

$$
[\mu]_{m}=\frac{(1)_{m}}{1-b_{m} \mu}
$$

and leads to

$$
[\mu]_{k}=\frac{(1)_{k} \sum_{l=1}^{m+1-k} a_{k+1}(l) \mu^{l-1}}{\sum_{l=1}^{m+2-k} a_{k}(l) \mu^{l-1}}, \quad(k=1,2, \cdots, m) .
$$

Here coefficients are

$$
\begin{gathered}
a_{k}(1)=1, \quad(k=1,2, \cdots, m+1), \\
a_{k}(2)=-\sum_{j=k}^{m} b_{j}, \quad(k=1,2, \cdots, m), \\
a_{m-1}(3)=-b_{m-1} a_{m}(2)-(1)_{m} .
\end{gathered}
$$

Furthermore

$$
a_{k}(l)=a_{k+1}(l)-b_{k} a_{k+1}(l-1)-(1)_{k+1} a_{k+2}(l-2),
$$

for $k=1,2, \cdots, m-2$ and $3 \leqq l \leqq m-k+1$, and

$$
a_{k}(l)=-b_{k} a_{k+1}(l-1)-(1)_{k+1} a_{k+2}(l-2),
$$

for $l=m-k+2$ where $k=1,2, \cdots, m-2$.

The poles $\left\{\mu_{q}^{(l)}\right\}$ with the $m$-pole approximation are therefore the roots of the order- $m$ algebraic equation

$$
\Omega_{m}(\mu) \equiv \sum_{l=1}^{m+1} a_{1}(l) \mu^{l-1}=0 .
$$

By putting $\nu=1 / \mu$, the equation $\Omega_{m}(1 / \nu)=0$ is therefore identical to the characteristic equation of (3.3) with the $m$-eigenstate approximation.

Let us comment on the validity of the finite-pole approximation of the continuedfraction expansion. It is obvious that the expansion (4.5) is meaningful only when $\left\{\left|b_{k}\right|\right\}$ 
are not too large. Assume that for one $k$-value, say $k=K(\leqq m),(1)_{K}$ vanishes (or, in a practical sense, is extremely small) for some $q$-value, $q=Q$. The quantity $\left|b_{K}\right|$ then diverges at $q=Q$, if $(2)_{K}$ remains finite. Since a part of $\Omega_{m}(\mu)$ contains terms $h(\mu) b_{K} \mu$ at $q=Q, h$ being a non-singular factor, the algebraic equation $\Omega_{m}(\mu)=0$ has a solution $\mu=0$ at $q=Q$, i.e one reciprocal eigenvalue $\nu$ diverges. As $q$ is changed from below $Q$, one reciprocal eigenvalue gradually, say, increases, and diverges at $q=Q$, i.e. $\nu=\infty$ for $q=Q-0$. For $q=Q+0, \nu=-\infty$. Near such points the continued-fraction expansion loses its validity.

\section{Example - Exact eigenvalues and finite-pole approximation -}

In this section we study a simple non-obvious solvable example and compare the finitepole approximation of the continued-fraction expansion with exact results. The model we discuss is the Markov map

$$
f(x)= \begin{cases}s_{0} x & (0 \leqq x \leqq a) \\ s_{1}(x-a) & (a<x \leqq 1)\end{cases}
$$

where $s_{0} \equiv a^{-1}$ and $s_{1} \equiv a /(1-a)$.

\section{A. Partition function and eigenvalues}

First, we calculate the partition function $Z_{q}(n)$ from periodic orbits. Let $I_{0}=[0, a]$ and $I_{1}=(a, 1]$ be the dynamical partition of the full interval $I=[0,1]$, i.e., the subintervals

on which $f$ has constant slope. $f$ maps $I_{0}$ onto $I$ and $I_{1}$ onto $I_{0}$. Higher iterates of $f$ give rise to finer subdivisions of $I$ which can be labelled by strings of letters $A$ and $B$, depending on whether the iterates come to lie in the subintervals $I_{0}$ or $I_{1}$ respectively. Since $I_{1}$ is mapped onto $I_{0}$, there can be no repetitions of the letter $B$, i.e., every $B$ is 
followed by an $A$. If the last letter of a string of length $k$ is $A$, then the $k$-th iterate of the subinterval is mapped onto the full interval and thus has a fixed point (cycle of period $k$ ). If the letter is a $B$, then there will be a fixed point only if $x \in I_{0}$.

The derivatives of $f^{(k)}$ at its fixed points are $s_{0}^{n_{A}} s_{1}^{n_{B}}$, where $n_{A}$ and $n_{B}$ are the number of intermediate points in the intervals $I_{0}$ and $I_{1}$, respectively. Since they depend only on the number and not on the ordering, periodic points with strings differing only in the ordering of symbols will give rise to the same derivatives and the same weights in the traces, eq. (3.11).

We now turn to the computation of the number of points with the same derivatives. To this end, we consider the sum of slopes, $W_{T}(k)$, over all fixed points of $f^{k}(x)$. A fixed point of $f^{k}(x)$ on a subinterval is classified into two groups $A$ and $B$ according to the last letter of the string. Let $W_{\sigma}^{A}(k),(\sigma=0$ or 1$)$ denote the sum of slopes over the fixed points in the group $A$ which are on $I_{\sigma}$. Similarly, $W_{\sigma}^{B}(k)$ is defined for the fixed points in the group $B$. Then, we can write the recursion equation

$$
\left.\begin{array}{l}
W_{0}^{A}(k)=s_{0}\left[W_{0}^{A}(k-1)+W_{0}^{B}(k-1)\right] \\
W_{0}^{B}(k)=W_{1}^{A}(k)=s_{1} W_{0}^{A}(k-1) \\
W_{0}^{A}(1)=s_{0}, \quad W_{0}^{A}(0)=0 .
\end{array}\right\}
$$

The sum $W_{T}(k)$ can be written as

$$
W_{T}(k)=W_{0}^{A}(k)+W_{0}^{B}(k)+W_{1}^{A}(k) .
$$

The recursion equation $(5.2 a)$ leads to

$$
W_{0}^{A}(k)=\frac{s_{0}}{y_{+}-y_{-}}\left[y_{+}^{k}-y_{-}^{k}\right],
$$

where we put

$$
y_{ \pm}=\frac{1}{2}\left[s_{0} \pm \sqrt{s_{0}^{2}+4 s_{0} s_{1}}\right] .
$$


Inserting (5.3) and (5.4) into (5.2b), we have

$$
\begin{aligned}
W_{T}(k) & =y_{+}^{k}+y_{-}^{k} \\
& =\left(\frac{1}{2}\right)^{k-1} \sum_{i=0}^{[k / 2]}\left(\begin{array}{c}
k \\
2 i
\end{array}\right) \sum_{j=0}^{i}\left(\begin{array}{l}
i \\
j
\end{array}\right)\left(4 s_{1}\right)^{j} s_{0}^{k-j},
\end{aligned}
$$

where [.] is Gauss's symbol. Because the slopes of $f^{k}(x)$ are written in the factorized forms $s_{1}^{j} s_{0}^{k-j}(j=0,1,2, \ldots,[k / 2])$, the number of the fixed points with the same slope is given by the corresponding coefficient of the homogeneous equation (5.5).

If we now specify the observable $u\{x\}$ to be the characteristic function on the interval $[a, 1]$, i.e.,

$$
u\{x\}= \begin{cases}0 & (0 \leqq x \leqq a) \\ 1 & (a<x \leqq 1)\end{cases}
$$

we can write the partition function (3.11) as

$$
Z_{q}(n)=\left(\frac{1}{2}\right)^{n-1} \sum_{i=0}^{[n / 2]}\left(\begin{array}{l}
n \\
2 i
\end{array}\right) \sum_{j=0}^{i}\left(\begin{array}{l}
i \\
j
\end{array}\right)\left(4 e^{q}\right)^{j} /\left(s_{1}^{j} s_{0}^{n-j}-1\right) .
$$

Now we evaluate eigenvalues of the operator $H_{q}$ with observable (5.6). As we are interested in an absolutely continuous measure, we consider the functional space $\mathcal{F}$ which consists of all linear combinations of polynomials on $I_{0}$ and $I_{1}$. Let $\chi_{\sigma},(\sigma=0$ or 1$)$ denote the characteristic function of $I_{\sigma}: \chi_{\sigma}(x)=1$ for $x \in I_{\sigma}$, and 0 for $x \notin I_{\sigma}$. Then, a linear combination $G \in \mathcal{F}$ is defined by

$$
G(x)=P_{j}(x) \chi_{0}(x)+P_{k}(x) \chi_{1}(x)
$$

where $P_{j}$ and $P_{k}$ are polynomials on $I$. Because the map (5.1) is piecewise linear and the $u\{x\}$ piecewise constant, the map $H_{q}: \mathcal{F} \rightarrow \mathcal{F}$,

$$
\left(H_{q} G\right)(x)=\left[s_{0}^{-1} P_{j}\left(s_{0}^{-1} x\right)+s_{1}^{-1} P_{k}\left(s_{1}^{-1} x+a\right) e^{q}\right] \chi_{0}(x)+s_{0}^{-1} P_{j}\left(s_{0}^{-1} x\right) \chi_{1}(x),
$$


preserves the maximal degree of polynominals. For $n=0,1,2, \ldots$, the set of linear combinations of polynomials of degree $k(0 \leqq k \leqq n)$ is a subspace of $\mathcal{F}$, denoted by $\mathcal{F}^{(n)}$. The restriction of $H_{q}$ onto $\mathcal{F}^{(n)}$ determines an operator $H_{q}^{(n)}: \mathcal{F}^{(n)} \rightarrow \mathcal{F}^{(n)}$. As a linear combination $G(x)$ in $\mathcal{F}^{(n)}$ can be represented by a $2(n+1)$-dimensional vector [11], and $H_{q}^{(n)}$ can be written as a $2(n+1) \times 2(n+1)$ matrix. Indeed, we have the upper-triangular matrix

$$
H_{q}^{(n)}=\left(\begin{array}{cccc}
h_{q}^{(0)} & & & * \\
& h_{q}^{(1)} & & \\
0 & & \ddots & \\
& & & h_{q}^{(n)}
\end{array}\right),
$$

where each element of $H_{q}^{(n)}$ is a $2 \times 2$ matrix and

$$
h_{q}^{(j-1)} \equiv\left(\begin{array}{cc}
s_{0}^{-j} & s_{1}^{-j} e^{q} \\
s_{0}^{-j} & 0
\end{array}\right), \quad(j=1,2, \ldots, n+1) .
$$

Eigenvalues of $h_{q}^{(j-1)}(1 \leqq j \leqq n+1)$ are also eigenvalues of $H_{q}^{(n)}$, and are given by

$$
t_{ \pm}^{(j-1)} \equiv \frac{1}{2}\left[s_{0}^{-j} \pm \sqrt{s_{0}^{-2 j}+4 e^{q}\left(s_{0} s_{1}\right)^{-j}}\right] .
$$

Since the full generalized Frobenius-Perron operator $H_{q}$ is obtained in the limit $n \rightarrow \infty$, the eigenvalues of $H_{q}$ are

$$
\nu_{q}^{(l)}= \begin{cases}t_{+}^{(j)} & \text { for } l \equiv 2 j=0,2,4, \ldots \\ t_{-}^{(j)} & \text { for } l \equiv 2 j+1=1,3,5, \ldots\end{cases}
$$

Substituting (5.12) into (3.14) and summing up over $l$ yields

$$
\operatorname{Tr} H_{q}^{n}=\left(\frac{1}{2}\right)^{n-1} \sum_{i=0}^{[n / 2]}\left(\begin{array}{l}
n \\
2 i
\end{array}\right) \sum_{j=0}^{i}\left(\begin{array}{l}
i \\
j
\end{array}\right)\left(4 e^{q}\right)^{j} /\left(s_{1}^{j} s_{0}^{n-j}-1\right) .
$$

We remark, that for this example the generating function (2.5) can be expressed in terms of eigenvalues of $H_{q}^{(0)}$ only as

$$
M_{q}(n)=\left\{\left[1-\nu_{q}^{(1)}\right]^{2}\left[\nu_{q}^{(0)}\right]^{n}-\left[1-\nu_{q}^{(0)}\right]^{2}\left[\nu_{q}^{(1)}\right]^{n}\right\} /\left[\nu_{q}^{(0)}-\nu_{q}^{(1)}\right]\left[2-\nu_{q}^{(0)}-\nu_{q}^{(1)}\right]
$$


B. Truncation of the continued-fraction expansion

We will study the approximate methods to obtain eigenvalues of the GFP operator for the model (5.1) by truncating the continued-fraction expansion. Hereafter we consider three approximations with 4-, 6-, and 8-poles, for which we need traces up to order $n=8$, 10 and 12 , respectively. The parameter is chosen as $a=0.75$.

Figure 1(a) shows the first twenty of the exact eigenvalues (5.13). Figures 1(b-d) show the comparison between results of the finite-pole approximations and exact results. In each approximation there exist apparent deviations of approximate values from the exact results in certain regions of $q$. Such deviations are located near $q$-values where one or several coefficients $(1)_{K}$ vanish, thus leading to a divergence of $\left|b_{k}\right|$ and a failure of the finite pole approximation of the continued fraction expansion. For the 4-pole approximation the region showing apparent deviation is rather wide. However, as the number of poles is increased, the number of such singular points increases, but the total area of effected $q$-regions diminishes. In this way the approximate results approach the exact ones as the number of poles is increased.

\section{Concluding remarks}

In closing this paper let us add several remarks. The first point concerns the relation between the characteristic function $\phi(q)$, especially for local expansion rates [13], and the decay rates $\left\{\gamma_{q}^{(l)}\right\}$ in $I_{q}(\omega)$. Whereas $\phi(q)$ describes the probability distribution of fluctuations, the decay rates $\left\{\gamma_{q}^{(l)}\right\}$ characterize the explicit temporal correlations. $\phi(q)$ is determined by the largest eigenvalue of $H_{q}$ and $\left\{\gamma_{q}^{(l)}\right\}$ by others [7]. In this sense, we conclude that decay rates generically have no relation with the characteristic function $\phi(q)$, 
except in special cases.

We proposed a continued-fraction expansion for the eigenvalues of the generalized Frobenius-Perron operator $H_{q}$, whose expansion coefficients are determined in terms of periodic orbits of the dynamical equation. A solvable illustrative example was presented, and their eigenvalues were compared with those of truncated continued-fraction expansions. It should be noted that whereas the largest eigenvalue is insensitive to the approximation (except in several narrow regions of $q$, related to the divergence of the coefficients $\left\{b_{k}\right\}$ ), other eigenvalues which are relevant to the temporal correlations depent rather sensitively on the order of truncation in the continued fraction expansion. Except for this, the finite pole approximation of the continued-fraction expansion turned out to be a powerful approximation.

The ordinary double time correlation function is determined by the eigenvalues of the FP operator $H$ [14]. The equations corresponding to (3.15) are

$$
\sum_{l}^{\prime}\left[\nu_{0}^{(l)}\right]^{n}=Z_{0}(n)-1
$$

where $\sum_{l}^{\prime}$ stands for the summation except $l=0$, because as a result of the existence of the invariant density the largest eigenvalue of $\left\{\nu_{0}^{(l)}\right\}$ is unity. As can be easily verified in our example, the eigenvalue $\nu_{q}^{(0)}=t_{+}^{(0)}$ (eq. (5.13)) indeed passes through 1 for $q \rightarrow 0$. In Ref. 3, eq (6.1) was solved approximately using the cycle expansion of Ruelle zeta functions and Fredholm determinants $d_{F}$. Because of possible poles in the Ruelle zeta functions, one has to use the full Fredholm determinant to determine the eigenvalues. As the calculations in appendix B show, in our solvable example, the Ruelle zeta functions are entire and the Fredholm determinant factorizes into a product of Ruelle zeta functions; thus there are no poles and the spectrum as computed from Ruelle zeta functions and the 
Fredholm determinant coincides. This exact factorization of course is not noticable from a truncated cycle expansion of the Fredholm determinant as used in numerical calculations. The numerically determined eigenvalues behave very much as in the case of the continued fraction expansion, except that they show no poles.

One might be lead to conclude from this example that the cycle expansion of appendix B seems to be better, but one can also find examples where the cycle expansion method has problems but where the continued fraction expansion works fine, e.g. in the case of the fully developped parabola. Thus the advantages and disadvantages of the two methods are more subtle, and certainly require further work.

As reported in $[7,8],\left\{\nu_{q}^{(l)}\right\}$ can be derived as the poles of the Laplace transform of $M_{q}(n)$, where we need the explicit expression of moments $M_{q}(1), M_{q}(2), M_{q}(3), \cdots$, using the explicit information on the natural invariant density itself. The present approach however does not need the natural invariant density to obtain $\left\{\nu_{q}^{(l)}\right\}$.

Finally let us comment on how the order of truncation $m$ should be chosen. Let $\tau$ and $\gamma$ be the characteristic periodicity and the damping rate, respectively. Depending on the type of chaotic transitions and on the observables, $\tau$ and $\gamma$ sometimes exhibit critical slowing-down. For example, for $x_{\mu}(j)$, one component of the orbit $\boldsymbol{x}(t)$ itself, the damping rate $\gamma$ tends to zero with finite $\tau$ as the control parameter approaches the band splitting point. And we find $\gamma \rightarrow 0$ and $\tau \rightarrow \infty$ near the type I intermittency. Although there are two characteristic time-scales $\tau$ and $\gamma^{-1}$, it is expected that $m$ should be chosen such that $m \gg \tau$, since $\tau$ is relevant to periodicity. (See Ref.[15].) 


\section{Appendix A - Derivation of (3.1) -}

Equation (3.1) can be obtained by noting that

$$
\begin{aligned}
M_{q}(n) & =\int \rho_{*}(\boldsymbol{x}) \exp \left[q \sum_{j=0}^{n-1} u\left\{\boldsymbol{f}^{j}(\boldsymbol{x})\right\}\right] d \boldsymbol{x} \\
& =\int \rho_{*}(\boldsymbol{x}) e^{q u\{\mathbf{x}\}} L \exp \left[q \sum_{j=0}^{n-2} u\left\{\boldsymbol{f}^{j}(\boldsymbol{x})\right\}\right] d \boldsymbol{x},
\end{aligned}
$$

where $L$ is the time evolution operator, $L G(\boldsymbol{x})=G(\boldsymbol{f}(\boldsymbol{x}))$. Since $\int G_{1}(\boldsymbol{x}) L G_{2}(\boldsymbol{x}) d \boldsymbol{x}=$ $\int\left[H G_{1}(\boldsymbol{x})\right] G_{2}(\boldsymbol{x}) d \boldsymbol{x}$, eq. (A.1) can be written as

$$
\int\left[H_{q} \rho_{*}(\boldsymbol{x})\right] \exp \left[q \sum_{j=0}^{n-2} u\left\{\boldsymbol{f}^{j}(\boldsymbol{x})\right\}\right] d \boldsymbol{x},
$$

where $H_{q}$ is the generalized Frobenius-Perron operator defined in (3.2).

By repeating the above procedure successively, $M_{q}(n)$ is written as

$$
\begin{aligned}
M_{q}(n)= & \int\left[H_{q}^{2} \rho_{*}(\boldsymbol{x})\right] \exp \left[q \sum_{j=0}^{n-3} u\left\{\boldsymbol{f}^{j}(\boldsymbol{x})\right\}\right] d \boldsymbol{x} \\
& \vdots \\
= & \int\left[H_{q}^{n-1} \rho_{*}(\boldsymbol{x})\right] \exp [q u\{\boldsymbol{x}\}] d \boldsymbol{x} .
\end{aligned}
$$

By noting $\int G(\boldsymbol{x}) d \boldsymbol{x}=\int H G(\boldsymbol{x}) d \boldsymbol{x}$, eq.(A.3) leads to eq.(3.1).

\section{Appendix B - Cycle expansion and dynamical zeta function -}

The cycle expansion approach to solving the moment problem (3.15) consists again in forming the Laplace transform (4.1) so as to obtain the eigenvalues as inverses of the positions of poles. However, this Laplace transform is then expressed as a logarithmic

derivative of a Zeta function, so that the eigenvalues are determined by zeros of this Zeta function. 
Consider again eq. (3.11), expressing the trace $Z_{q}(n)$ in terms of periodic orbits. Let $p$ label all primitive cycles of period $n_{p}\left(n_{p}\right.$ is the smallest number of iterations after which the orbit closes). Note that both

$$
u_{p}=\sum_{j=1}^{n-1} u\left\{f^{j}\left(x^{(n), k}\right)\right\}
$$

and

$$
\Lambda_{p}=\left|D_{n}\left(\boldsymbol{x}^{(n), k}\right)\right|
$$

are independent of the point where they are computed. A cycle of period $n_{p}$ has $n_{p}$ different periodic points. In the sum (3.11), it contributes whenever $k=n_{p}$ or a multiple thereof. Thus when summing on all periods in the Laplace transform (4.11), viz.

$$
Z_{q}[\mu]=\sum_{n=1}^{\infty} Z_{q}(n) \mu^{n-1}
$$

one can also express this as a sum on all primitive periodic orbits and their multiple traversals,

$$
Z_{q}[\mu]=\sum_{p} \sum_{r=1}^{\infty} n_{p} \frac{\exp \left[q r u_{p}\right]}{\left|1-\Lambda_{p}^{r}\right|} \mu^{r n_{p}-1} .
$$

The usual manipulations (expansion of the denominator in a geometric series and summation on $r)[3]$ result in

$$
Z_{q}[\mu]=-\left(\frac{\partial}{\partial \mu}\right) \log \Omega(\mu)
$$

where the Zeta function

$$
\Omega(\mu)=\prod_{p} \prod_{j=0}^{\infty}\left(1-\mu^{n_{p}} \exp \left(q u_{p}\right)\left|\Lambda_{p}\right|^{-1} \Lambda_{p}^{-j}\right)=\prod_{j=0}^{\infty} \zeta_{j}^{-1}
$$

factorizes into dynamical zeta functions $\zeta_{j}^{-1}$. As we will see, for the particular map studied in section 5, each dynamical zeta function will be a quadratic polynominal and thus contribute two eigenvalues for each $j$. 
The key to the evaluation of the products on periodic orbits is the topological organization of the periodic orbits. In the example of section 5 , there can be no two consequtive $B$ 's, since every point in the interval $[a, 1]$ is mapped back into the interval $[0, a]$. This is the only exclusion rule. Defininig a new alphabet $0 \equiv A$ and $1 \equiv B A$, one finds that all strings of 0 and 1 can be realized. Since moreover the maps is linear, all weights $\Lambda_{p}$ factorize according to

$$
\Lambda_{p}=\Lambda_{A}^{n_{0}} \Lambda_{A B}^{n_{1}}
$$

where $n_{0}$ and $n_{1}$ are the numbers of 0 's and 1's in the symbol string of $p$, respectively. The instabilities $\Lambda_{p}$ are

$$
\Lambda_{A}=s_{0}, \quad \Lambda_{A B}=s_{0} s_{1},
$$

and the observable (5.6) becomes

$$
u_{p}=n_{1} .
$$

One can then show $[3,16]$ that the dynamical zeta functions become

$$
\zeta_{j}^{-1}=1-\mu\left|s_{0}\right|^{-1} s_{0}^{-j}-\mu^{2} \exp [q]\left|s_{0} s_{1}\right|^{-1}\left(s_{0} s_{1}\right)^{-j} .
$$

which is precisely the characteristic polynominal for the submatrices $(5.11), \zeta_{j}^{-1}=\operatorname{det}(1-$ $\mu h_{q}^{(j)}$ ) for $j=0,1, \cdots$. Thus in this case the cycle expansion yields the exact eigenvalues. 


\section{References}

[1] See, e.g.,

Sinai, Ya. G.: Uspekhi Math. Nauk 27, 27 (1972)

Bowen, R.: Equilibrium States and the Ergodic Theory of Anosov Diffeomorphisms, Lecture Notes in Mathematics 470 (New York: Springer-Verlag, 1975)

Ruelle, D.: Thermodynamic Fromalism in Encyclopedia of Mathematics and Its Applications, Vol.5, (Addison-Wesley, Reading, 1978)

[2] See, e.g., the following articles:

Kai, T., Tomita, K.: Prog. Theor. Phys. 64, 1532 (1980)

Takahashi, Y., Oono, Y.: Prog. Theor. Phys. 71, 851 (1984)

Auerbach, D., Cvitanovic, P., Eckmann, J.-P., Gunaratne, G., Procaccia, I.: Phys.

Rev. Lett. 58, 2387 (1987)

Morita, T., Hata, H., Mori, H., Horita, T., Tomita, K.: Prog. Theor. Phys. 296 (1988)

Cvitanovic, P.: Phys. Rev. Lett. 61, 2729 (1988)

Cvitanovic, P., Gunaratne, G. H., Procaccia, I.: Phys. Rev. A 38, 1503 (1988)

[3] Cvitanović, P., Eckhardt, B.: J. Phys. A: Math. Gen. 24, L237 (1991)

Christiansen, F., Paladin, G., Rugh, H. H.: Phys. Rev. Lett. 65, 2087 (1990)

Rugh, H. H.: Nonlinearity 5, 1237 (1992)

Eckhardt, B.: Acta Phys. Pol. B 24, (1993) at press

[4] Fujisaka, H., Inoue, M.: Prog. Theor. Phys. 77, 1334 (1987) 
[5] Fujisaka, H., Inoue, M.: Prog. Theor. Phys. 78, 268 (1987)

[6] Fujisaka, H.: in From Phase Transitions to Chaos (Topics in Modern Statistical Physics), eds. Györgyi, G., Kondor, I., Sasvári, L., and Tél, T., (World Scientific, 1992)

[7] Fujisaka, H., Shibata, H.: Prog. Theor. Phys. 85, 187 (1991) Just, W., Fujisaka, H.: Physica D (1993), at press

[8] Fujisaka, H., Inoue, M.: Prog. Theor.Phys. 78, 1203 (1987)

[9] Just, W.: J. Stat. Phys. 67, 271 (1992)

[10] Ellis, R. S.: Entropy, Large Deviations, and Statistical Mechanics, (New York: Springer-Verlag, 1985)

[11] See, e.g., the following articles:

Grossmann, S., Thomae, S.: Z. Naturforsch. 32a, 1353 (1977)

Mori, H., So, B. C., Ose, T.: Prog. Theor. Phys. 66, 1266 (1981)

Shigematsu, H., Mori, H., Yoshida, T., Okamoto, H.: J. Stat. Phys. 30, $649(1983)$

[12] Szépfalusy, P., Tél, T.: Phys. Rev. A 34, 387 (1986)

[13] Badii, R., Heinzelmann, K., Meier, P. F., Politi, A.: Phys. Rev. A 37, $1323(1988)$

[14] Mori, H., et al. and Shigematsu, et al. in Ref. [11]

[15] Just, W., et al. in Ref.[7]

Kobayashi, T., Fujisaka, H., Just, W.: Phys. Rev. E, (1993) at press Just, W., et al., to be submitted

[16] Artuso, R., Aurell, E., Cvitanović, P.: Nonlinearity 3, 325 and 361 (1990) 
Cvitanović, P., Eckhardt, B.: Nonlinearity, (1993), at press 


\section{Figure Caption}

Fig.1 (a) Exact eigenvalues of $H_{q}$ for the model (5.1) with $a=0.75$. Twenty eigenvalues vs $q$ are shown in the order of increasing values. The results from finite-pole approximations of the continued-fraction expansion are shown in figures (b) using 4 poles, (c) using 6 poles and (d) using 8 poles. Solid and dotted lines respectively indicate results of finite-pole approximation and exact results. As the number of poles is increased, the results tend to agree with the exact results. 